\title{
Study of Three-dimensional Display System Based on Computer-generated Integral Photography
}

\author{
Sungyong Jung, Sung-Wook Min, Jae-Hyeung Park, and Byoungho Lee* \\ National Research Laboratory of Holography Technologies \\ School of Electrical Engineering, Seoul National University, Seoul 151-744, KOREA
}

(Received December 5, 2000)

\begin{abstract}
A three-dimensional (3D) display system based on computer-generated integral photography (CGIP) is proposed and its feasibility is discussed. Instead of the pickup process in conventional IP, the elemental images of imaginary objects are computer-generated. Using these images, we observed autostereoscopic 3D images in full color and full parallax. The lateral and depth resolutions of the integrated images are limited by some factors such as the image position, object thickness, the lens width, and the pixel size of display panel.
\end{abstract}

OCIS codes : $110.2960,110.4850,120.2040,220.4830$.

\section{INTRODUCTION}

A variety of approaches have been investigated to display three-dimensional (3D) images at high resolution and a wide viewing angle. Binocular stereoscopic display, autostereoscopic display, and holographic display techniques represent typical methods for creating $3 \mathrm{D}$ images and each method has its own advantages and disadvantages. Integral photography (IP), which provides a continuous viewpoint and does not require the use of special glasses, is one of the most attractive methods for autostereoscopic display. The IP method, the principle of which is shown in Fig. 1, and which is based on a photographic plate was first proposed by Lippmann in 1908 [1]. A lens sheet consisting of many elemental lenses is placed between the object and the photographic plate or film. A number of slightly different pictures of the object, observed from the various directions, are recorded on the film. The film is then developed and placed at exactly the same relative position. For the reproduction process, the film is irradiated with a diffused light. The rays retrace the original routes and reproduce the image at the position where the object was located. An autostereoscopic image is, thus, obtained. However this method is not suited for 3D animated images because of the film pickup process.

To overcome this problem, a method which involves the use of a CCD camera and a display panel for pickup and reconstruction, respectively, has been proposed. Pickup images for moving objects can be ob- tained in two ways. The first method involves the use of a diffuser or screen on which focused elemental images are displayed and captured by a CCD camera (indirect pickup) [2]. The major problem with this method is the loss of light. This loss degrades the photosensitivity and the resolution of the pickup images. The other method is called direct pickup. In this method, a large-aperture convex lens is inserted instead of a diffuser [3]. As a result, a group of elemental images created by a lens array is taken directly by a CCD camera. This method has some advantages compared with the indirect pickup. Nonetheless, both methods are two-step processes (pickup-reproduction) and include inevitable limitations in spatial resolution. Resolution is limited by the number of pixels for each elemental image in the pickup (as well as in the display) device.

This paper describes a computer-generated IP (CGIP) for 3D display and discusses some basic experimental results. In the CGIP procedure, the pickup process is not necessary because the pickup images are replaced by computer-generated or computer-assisted images.

\section{COMPUTER-GENERATED INTEGRAL PHOTOGRAPHY}

A major problem in the conventional IP scheme is the pseudoscopic reconstructed image. This is due to the fact that the viewers observe the image from the 


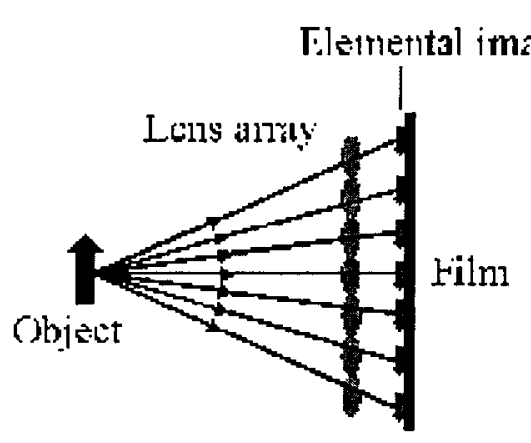

(a)

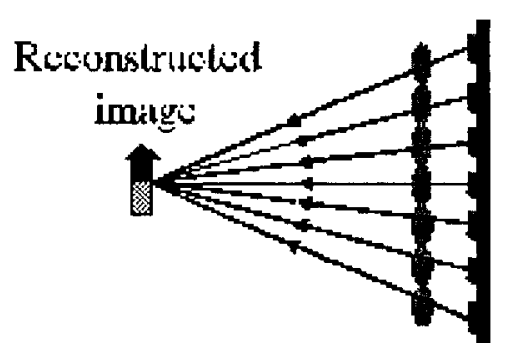

(b)

FIG. 1. Conventional integral photography (a) Pickup (b) Reconstruction.

opposite side of the pickup image. Therefore, a convex object is observed when a concave object is captured, and vice versa. Fig. 2 shows the concept of the orthoscopic and pseudoscopic images. This pseudoscopicorthoscopic image conversion performed by optical or electrical methods is required. To correct the problem of reversed depth, each captured elemental image must be rotated centrosymmetrically before being displayed on the display panel. Several methods to convert pseudoscopic images have been proposed by a number of workers. A conversion using a series of lens arrays [?], referred to as two-step IP, or a prism array was performed, but complex lens systems are required for this procedure. Recently IP using a graded-index (GRIN) lens-array has been reported as a reliable method that prevents pseudoscopic images [6]. Because the GRIN lens has a parabolic index profile, the trajectories of the rays within it are self-focused. If a GRIN lensarray with a proper length is used during pickup, the elemental images are not inverted.

In the CGIP system proposed in this paper, no 3D image pickup system is involved, because all elemental images for the display are processed by computer. Fig. 3 shows a basic schematic diagram of the CGIP. Computer-generated elemental images are displayed on the display panel. A lens array for image integration is placed in front of the panel. The distance between the lens array and the panel is close to the focal length of the lens array. In the case of CGIP, in-

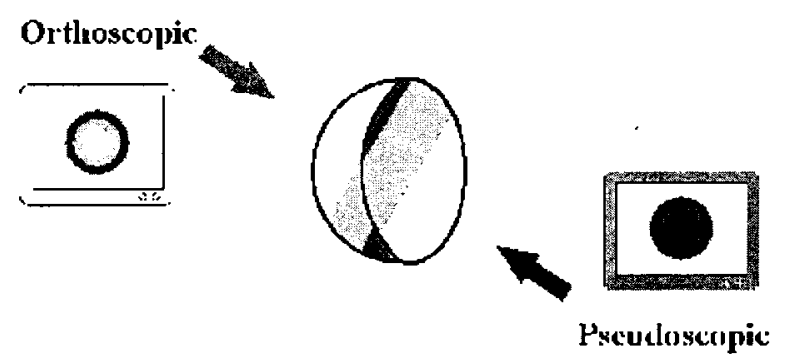

FIG. 2. The concept of the orthoscopic and psedoscopic images. stead of elemental pickup images, elemental images of the desired imaginary object are calculated. We assume an imaginary object which contains 3D information. If we define the lateral plane parallel to the display panel as the $x-y$ plane, the $z$ direction represents the information relative to depth. An imaginary object includes information relative to $x, y, z$ and each elemental image on the display panel has $x, y$ data. Therefore, if we generate calculated elemental images which correspond to a point of the imaginary object, the $3 \mathrm{D}$ information is mapped to an array of $2 \mathrm{D}$ information. We refer to this process as image mapping. Image mapping is performed point by point considering depth ( $z$-direction) information. In addition, the calculated elemental images are made orthoscopic. In this way, the problem of pseudoscopic images can be solved naturally. Fig. 4 shows the flow chart of the image mapping calculation. First, the center of each elemental lens (in a lens array) is calculated. Then the imaginary object is modeled as the combination of planes with different depths, which are arranged along the depth direction. For a point of an object, a set of elemental image points can be obtained. The procedure is as follows. Information of the $x-y$ coordinates of a point in an imaginary object determine the center of corresponding elemental image points. Based on information of the $z$ coordinate of an ima-

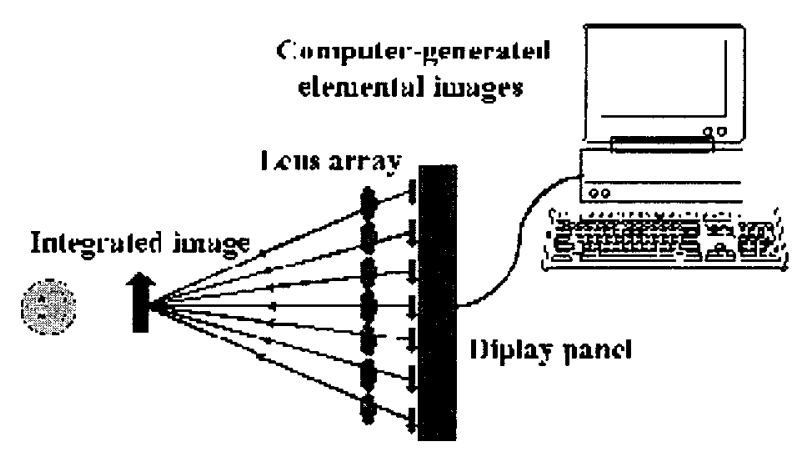

FIG. 3. Schematics of the CGIP system. 


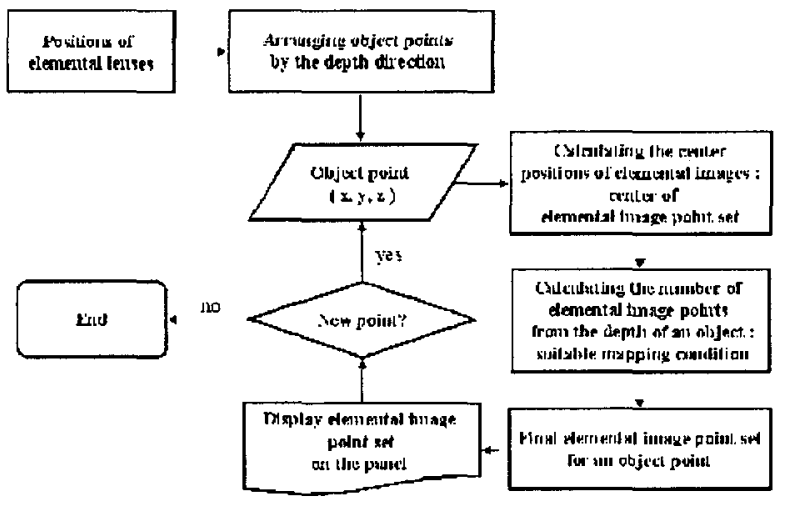

FIG. 4. Flow chart for image mapping.

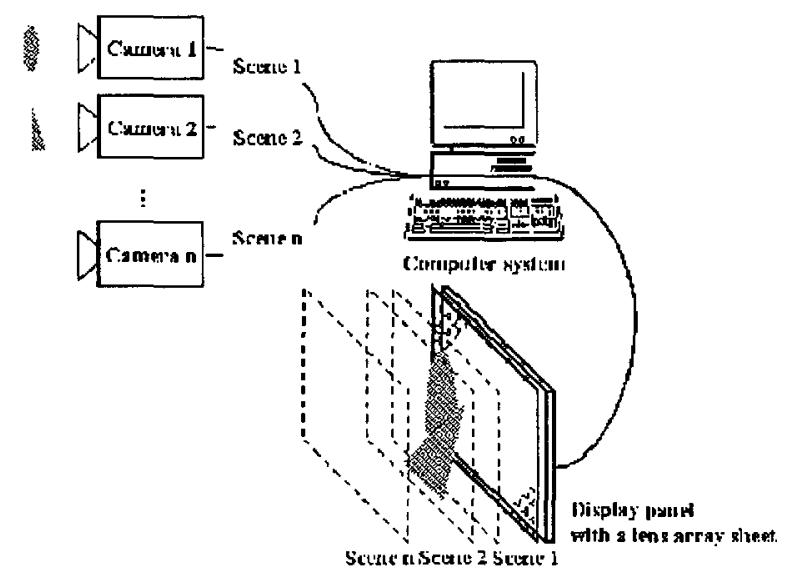

FIG. 5. Schematics of the quasi-3D display system.

ginary object, the positions and the number of elemental images can be calculated and, as a result, the set of elemental image points can be plotted. This calculation process is performed repeatedly until the set of elemental images for all points of an imaginary object are determined.

The CGIP scheme can also be applied to the quasi3D display system. Fig. 5 shows the schematics of the quasi-3D display system. If each camera picks a scene which is a part of the total view, and elemental images are computer-generated so that each scene has different depth, then a real object captured by an or-

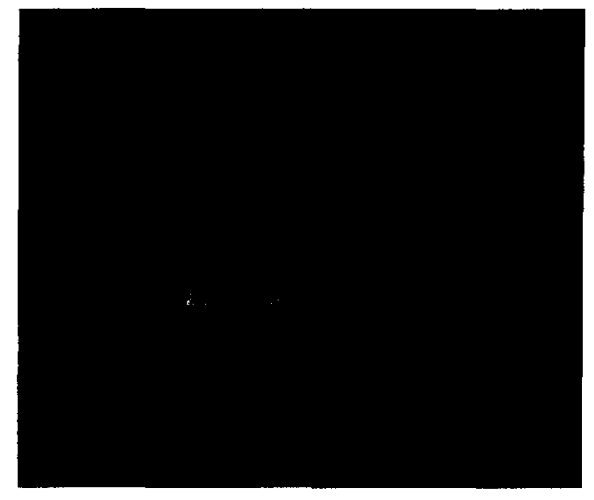

FIG. 6. CGIP elemental images of two spheres.

dinary camera can be displayed in quasi-3D. Therefore, a 3D display system without the lens array pickup system is possible. Of course, if we use an imaginary object which contains complete 3D information, the true $3 \mathrm{D}$ image as well as the quasi-3D image can be obtained.

Some basic experiments using the CGIP system have been performed. Table 1 shows the specifications of the image generation setup. The shape of each elemental lens is square and the pitch is the same as the lens width. The number of elemental images is $13(\mathrm{H}) \times 13(\mathrm{~V})$, which is not sufficient for commercial use, but the concept of CGIP can be verified with this system. The viewing angle is determined by the relation of the width and the focal length of the lens in the lens array. The calculated viewing angle of the system is about 10 degrees horizontally and vertically.

Fig. 6 shows elemental images of two spheres which have different depths. The depth means the distance from the lens array to the integral image position. Fig. 7 shows the integrated 3D images. The depth of the right sphere is larger than that of the left sphere and the depth difference is $8 \mathrm{~cm}$. The gap between the lens array and the LCD panel is determined by the depth of an object. The images from a left and a right viewpoint are shown, which clearly indicate that different 3D images are obtained from different viewpoints. This fact proves that the $3 \mathrm{D}$ images are autostereoscopic with full parallax. The 3D animated object was also displayed in real time successfully.

TABLE 1. Specifications for the image integration setup.

\begin{tabular}{lcc}
\hline \hline & Number of pixels & $640(\mathrm{H}) \times 480(\mathrm{~V})$ \\
$125 \times 95 \mathrm{~mm}^{2}$ \\
Display panel & Size of display panel & $65 \times 65 \mathrm{~mm}^{2}$ \\
& Active display area & $13(\mathrm{H}) \times 13(\mathrm{~V})$ \\
Lens array & Number of elemental lenses & $5 \mathrm{~mm}$ \\
& Pitch of the elemental lens & $30 \mathrm{~mm}$ \\
\hline \hline
\end{tabular}




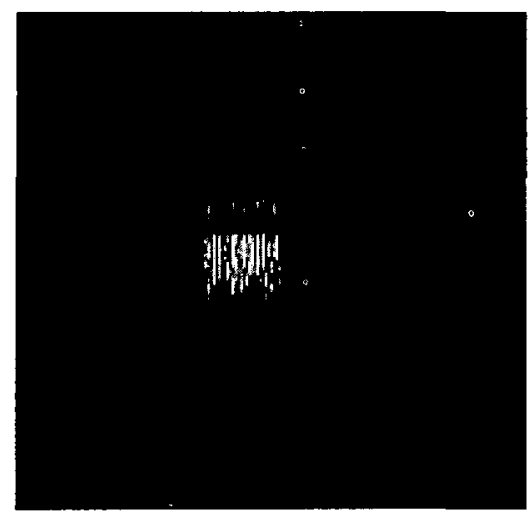

(a)

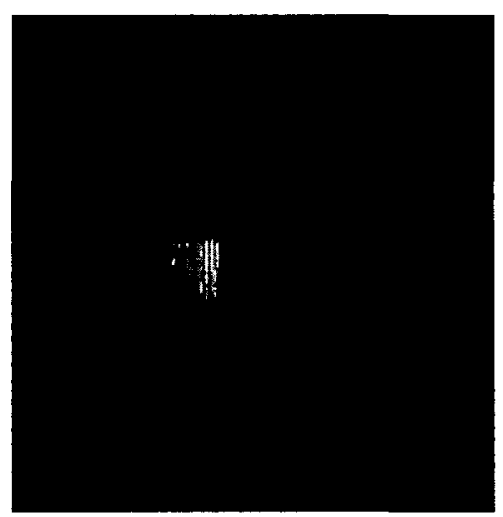

(b)

FIG. 7. Autostereoscopic 3D images (a) From the left view point (b) From the left view point.

Fig. 8 shows an example of quasi-3D display system. Though the object pictures are computer graphic images in this experiment, a real captured image is also available. The Integrated image is composed of three image planes with different depths: $9 \mathrm{~cm}, 10 \mathrm{~cm}, 11 \mathrm{~cm}$, respectively. The resolution of the integrated image is not high enough, but we can observe a quasi-3D image. If the resolution and the viewing angle of the system are improved, this method might be applied to a 3D TV system.

\section{RESOLUTION LIMITATIONS OF CGIP}

The resolution of the integrated image is classified into two types: The lateral resolution which represents the resolution of the integrated image at a given image plane and the longitudinal(depth) resolution which is related to the number of image planes into the depth direction. Fig. 9 shows the basic geometry of CGIP. It is assumed that an imaginary object with thickness $\mathrm{T}$ is displayed. The central plane of the object is loca-

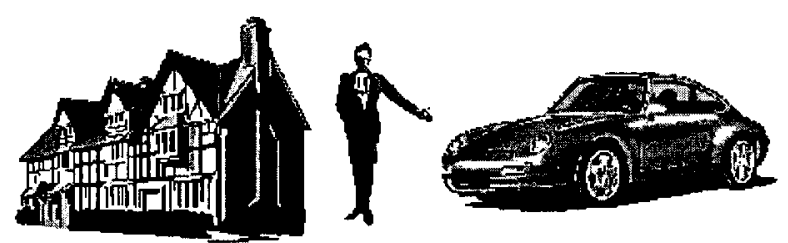

(a)

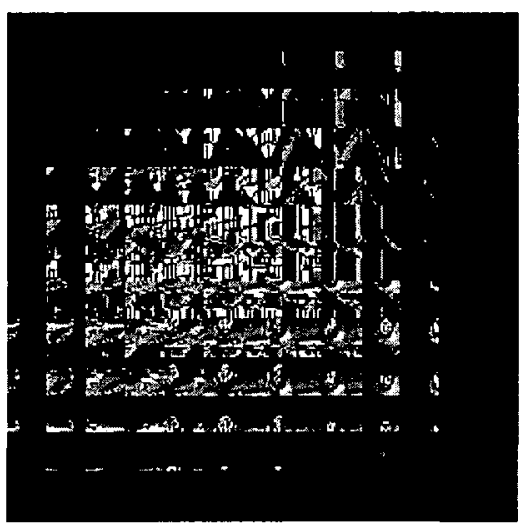

(b)

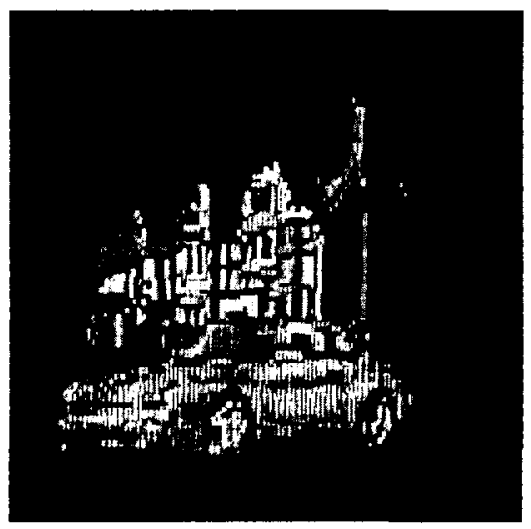

(c)

FIG. 8. Example of quasi-3D display system (a) Object pictures of quasi-3D display (b) Elemental images (c) Integrated image. 


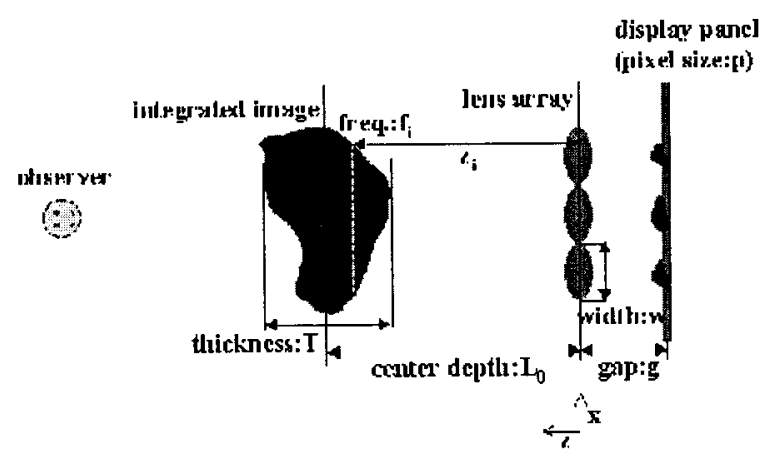

FIG. 9. Basic geometry of CGIP.

ted a distance $L_{0}$ away from elemental lenses with width $w$. The gap between the lens array and the display panel is $g$. The focal length of the elemental lens is assumed to be $f$. If we define the lateral resolution $f_{i}$ as the spatial resolution of the image at plane $z=z_{i}, f_{i}$ can be obtained by calculating the cutoff frequency of the modulation transfer function(MTF) of the lens. Under incoherent illumination, the MTF is expressed as follows.

$$
M T F=\left\{1-\frac{\lambda f_{i} z_{i}}{w}\right\} \operatorname{sinc}\left[w\left\{1-\frac{\lambda f_{i} z_{i}}{w}\right\} f_{i} z_{i} \operatorname{err}\left(z_{i}\right)\right]
$$

where

$$
\operatorname{err}\left(z_{i}\right)=\left|\frac{1}{z_{i}}+\frac{1}{g}-\frac{1}{f}\right|
$$

In this case, if the gap $g$ is adjusted to minimize the focusing error of the central image plane, $\operatorname{err}\left(z_{i}\right)$ is expressed as follows.

$$
\operatorname{err}\left(z_{i}\right)=\left|\frac{1}{z_{i}}-\frac{1}{L_{0}}+e r r_{\min }\right|
$$

where

$$
e r r_{\min }=\frac{1}{L_{0}}+\frac{1}{g}-\frac{1}{f}
$$

Eq. (1) contains both the effect of diffraction limitation due to the lens width and the effect of the focusing error. Therefore, if the lens width is too small, the lateral resolution of the integrated image is limited by diffraction. If the lens width is too large, the resolution is limited by the focusing error. Here we assume that the lens width is large enough to neglect the effect of diffraction. i.e., $w^{2} \operatorname{err}\left(z_{i}\right) \gg \lambda$. Hence, the cutoff frequency of the MTF is approximated as follows.

$$
f_{c} \approx \frac{1}{w z_{i} \operatorname{err}\left(z_{i}\right)}
$$

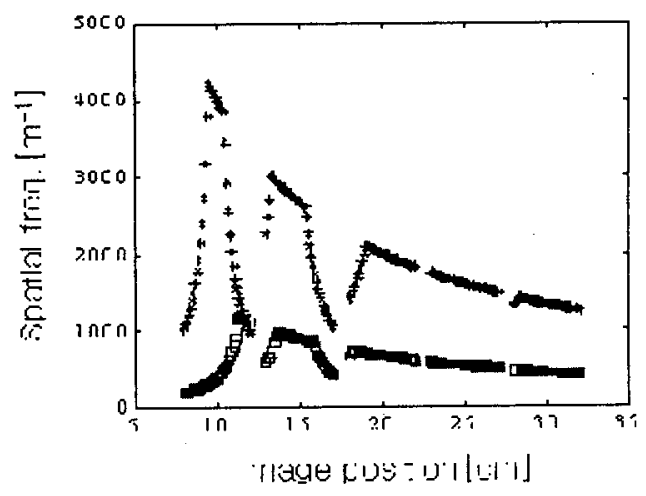

FIG. 10. Lateral spatial resolution.

From Eqs. (3) and (5), it is indicated that the lateral resolution of the integral image is degraded at image positions far from the lens array. We also see that the focusing error increases at a distance far from the central plane of the object. This means that the thickness of object also limits the resolution of an integrated image.

Fig. 10 shows the relation between the image position $z_{i}$ and the lateral resolution. The object thickness is assumed to be $4 \mathrm{~cm}$. So the range of the integrated image position is $2 \mathrm{~cm}$ before and behind the center depth $L_{0}$. The lateral resolution at image position $z_{i}$ is simulated when the center depth $L_{0}$ is $10 \mathrm{~cm}, 15 \mathrm{~cm}$, $20 \mathrm{~cm}, 25 \mathrm{~cm}, 30 \mathrm{~cm}$. The star line corresponds to the lens width of $5 \mathrm{~mm}$ and the square line corresponds to $15 \mathrm{~mm}$. From the figure, the resolution of the image produced near the lens array is higher but degraded at the image position around the center depth. Another factor limiting the lateral resolution is the finite pixel size of the display panel. The point of the integral image spreads in proportion to the pixel pitch $p$ and

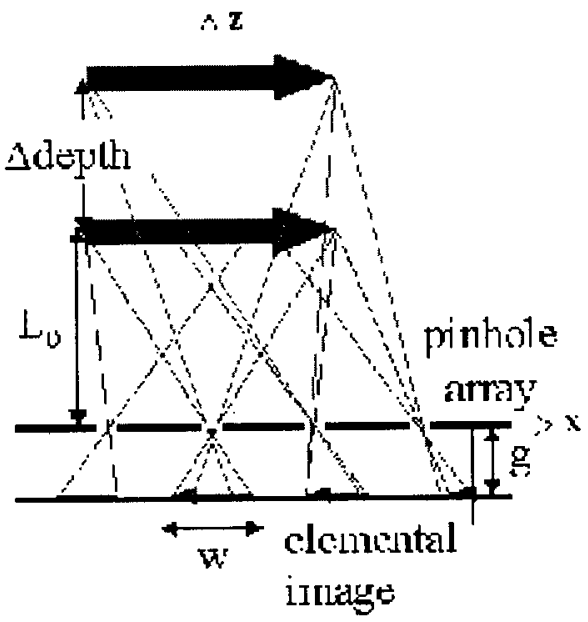

FIG. 11. Geometry for calculating depth resolution. 


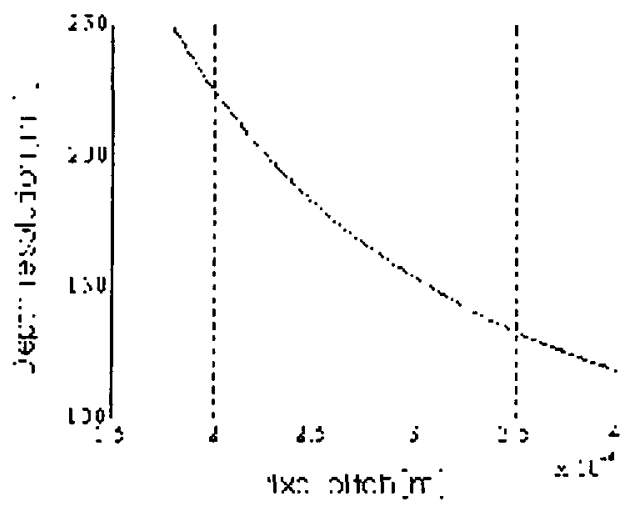

(a)

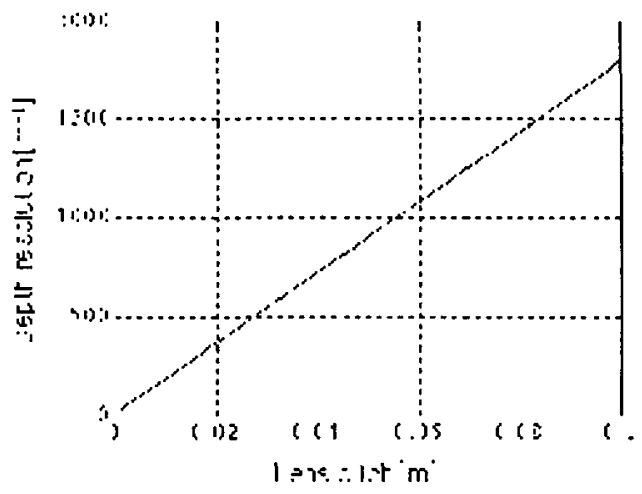

(b)

FIG. 12. Depth resolution (a) Resolution versus the pixel pitch (b) Resolution versus the lens pitch.

the image position $z_{i}$. From geometrical optics, we can write

$$
f_{i} \propto \frac{g}{p z_{i}} .
$$

Therefore, if we want to obtain good 3D image quality in CGIP system, it is indispensable to use a high resolution display panel and to limit the depth and thickness of an imaginary object.

The depth resolution means the number of resolvable image planes in longitudinal direction. Fig. 11 shows the simple geometry for calculating the depth resolution. Assuming the lens array to be a pinhole array, elemental images of striped arrows integrate an image with depth $L_{0}$. To display an integral image at another image plane, the shape of elemental images must be changed. However, the change is limited due to the finite pixel size of the display panel. If the length of the elemental image decreases by the pixel pitch $p$, the depth of an integral image increases by $\Delta$ depth, which is the minimum change of depth. The depth resolution $f_{d}$, is the inverse of $\Delta$ depth, and is expressed as follows.

$$
f_{d}=\frac{1}{\Delta d e p t h}=\frac{1}{\left|\frac{g w}{p+g w / L_{0}}-L_{0}\right|} .
$$

Fig. 12 shows the relation between the depth resolution and the pixel and lens pitch when other parameters are fixed. As we expected, the depth resolution is proportional to the lens width and inversely proportional to the pixel size of the display panel.

\section{CONCLUSION}

In this paper, a CGIP system is proposed and its feasibility is verified via some basic experiments. This method can be used in displaying 3D animation without the need for any special glasses. Generated autostereoscopic 3D images could be viewed in full color and full parallax. 3D animated images were also observed. The lateral and depth resolution limitations of CGIP were also analyzed. If the resolution and the viewing angle of the system are improved, the CGIP might be applied to a 3D TV system.

*Corresponding author : byoungho@plaza.snu.ac.kr

\section{REFERENCES}

[1] G. Lippmann, Comptes-Rendus 146, 446 (1908).

[2] M. McCormick, Proc. 2nd Int. Display Workshop, (ITE, Tokyo, 1995),77.

[3] F. Okano, H. Hoshino, J. Arai, and I. Yuyama, Appl. Opt. 36, 1598 (1997).

[4] H. E. Ives, J. Opt. Soc. Am. 21, 171 (1931).

[5] R. L. DeMontebello, Proc. SPIE 120, 73 (1977).

[6] J. Arai, F. Okano, H. Hoshino, and I. Yuyama, Appl. Opt. 37, 2034 (1998). 